\title{
Some New Properties of the Triangle.
}

By J. S. Mackay, M.A., I.L.D.

[The substance of this communication will be included in $\mathrm{Dr}$

Mackay's paper on The Triangle in the first volume of the Proceedings, which is about to be printed.]

\section{Proofs of some optical theorems. \\ By William Peddie, D.Sc.}

[The results of this paper will be contained in Dr Peddie's book on Physics, which will appear in a short time.]

Second Meeting, December 12th, 1890.

R. E. Allardice, Esq., President, in the Chair.

On the condition that the straight line

$$
l x+m y+n z=0
$$

should be a normal to the conic

$$
(a, b, c, f, g, h)(x, y, z)^{2}=0
$$

the co-ordinates being trilinear.

\section{By R. H. Pinkerton, M.A.}

1. The condition in question may be found by using the following theorem :-

If the equation in trilinear co-ordinates

$$
\mathrm{F}(x, y, z) \equiv\left(u, v, w, u^{\prime}, v^{\prime}, w^{\prime}\right)(x, y, z)^{2}=0 \quad . .
$$

represents a pair of straight lines, then the line whose equation is

$$
l x+m y+n z=0 \quad \ldots \quad \ldots \quad \ldots
$$

will be perpendicular to one of those lines if $\mathrm{F}(l-m \cos \mathrm{C}-n \cos \mathrm{B}, m-n \cos \mathrm{A}-l \cos \mathrm{C}, n-l \cos \mathrm{B}-m \cos \mathrm{C})=0$ where $\mathrm{A}, \mathrm{B}, \mathrm{C}$ are the angles of the fundamental triangle. 